\title{
Carta ao Editor: Reconstrução ligamentar da membrana interóssea do antebraço, no tratamento da instabilidade da articulação radioulnar distal
}

\section{Letter to the Editor: Ligamentous Reconstruction of the Interosseous Membrane of the Forearm in the Treatment of Instability of the Distal Radioulnar Joint}

\author{
Stavros Angelis ${ }^{1,2}$ Spyridon Triantafyllou ${ }^{1}$ Panagiotis Skandalakis ${ }^{1}$ Dimitrios Filippou ${ }^{1}$
}

\author{
${ }^{1}$ Departamento de Anatomia e Anatomia Cirúrgica, Faculdade de \\ Medicina, National and Kapodestrian University of Athens, Atenas, \\ Grécia \\ 2 Departamento de Ortopedia, Korgialenio-Benakio Hellenic Red \\ Cross Hospital, Atenas, Grécia
}

Rev Bras Ortop 2020;55(3):386.

Caro Editor,

Recentemente, lemos um artigo muito interessante sobre uma nova abordagem no tratamento da instabilidade longitudinal da articulação radioulnar distal (ARUD), publicada por Aita et al. Rev Bras Ortop. 2018;53(2): 184-191. Os resultados da abordagem proposta parecem ser muito promissores, enquanto que a técnica parece ser relativamente simples, viável e rápida, com uma curva curta de aprendizado. Todas as vantagens acima mencionadas sugerem que esta pode representar uma excelente opção de tratamento alternativo.

Segundo os autores, a técnica utiliza o tendão do músculo braquiorradial para passá-lo através de um túnel radial e ulnar, de forma oblíqua, proximal no rádio e distal na ulna, sendo fixado com um parafuso de bloqueio em cada osso. Isto também é demonstrado nas Figs. 3, 4 e 5. De acordo com Noda et al., ${ }^{1}$ quem primeiro relatou e chamou de feixe oblíquo distal (DOB, na sigla em inglês) em 2009, “o DOB se originou mais ou menos a partir de um sexto da área distal da diáfise ulnar, coincidindo aproximadamente com a borda proximal do músculo pronador quadrado, correndo distalmente em direção à ARUD. As fibras se misturaram ao tecido capsular da ARUD e, por fim, o DOB se insere na borda inferior do entalhe sigmóide do rádio."

Os autores apresentaram sua abordagem como sendo uma técnica de reconstrução do DOB, porém isso parece não ser preciso, já que o curso do enxerto do tendão braquiorradial está exatamente oposta ao curso normal do DOB. O curso que
Endereço para correspondência Stavros Angelis, PhD, Department of Anatomy and Surgical Anatomy, Medical School, National and Kapodistrian University of Athens, and Department of Orthopedics, Korgialenio-Benakio Hellenic Red Cross Hospital, 75 Mikras Asias Street, GR-11527 Goudi, Athens, Greece (e-mail: sangelis@hotmail.gr).

os autores descrevem tem maior probabilidade de representar o curso do "trato" da membrana interóssea descrito por Gabl et al. ${ }^{2}$ Em nossa opinião, a técnica de reconstrução que melhor simula o DOB é a descrita por Riggenbach et al. ${ }^{3}$

O DOB é uma estrutura da membrana interóssea distal, parecendo ser um importante estabilizador isométrico para a ARUD, de acordo com Moritomo et al. ${ }^{4}$ Seu significado anatômico e clínico ainda está em avaliação, portanto, descrições precisas e cuidadosas são de grande importância.

Conflito de Interesses

Os autores declaram não haver conflito de interesses.

\section{Referências}

1 Noda K, Goto A, Murase T, Sugamoto K, Yoshikawa H, Moritomo H. Interosseous membrane of the forearm: an anatomical study of ligament attachment locations. J Hand Surg Am 2009;34(03):415-422

2 Gabl M, Zimmermann R, Angermann P, et al. The interosseous membrane and its influence on the distal radioulnar joint. An anatomical investigation of the distal tract. J Hand Surg $[\mathrm{Br}]$ 1998; 23(02):179-182

3 Riggenbach MD, Conrad BP, Wright TW, Dell PC. Distal oblique bundle reconstruction and distal radioulnar joint instability. JWrist Surg 2013;2(04):330-336

4 Moritomo H, Noda K, Goto A, Murase T, Yoshikawa H, Sugamoto K. Interosseous membrane of the forearm: length change of ligaments during forearm rotation. J Hand Surg Am 2009;34(04):685-691 recebido

19 de Novembro de 2018 aceito

18 de Dezembro de 2018
DOI https://doi.org/ 10.1055/s-0039-1700831. ISSN 0102-3616.
Copyright $\odot 2020$ by Sociedade Brasileira License terms de Ortopedia e Traumatologia. Published by Thieme Revinter Publicações Ltda, Rio de Janeiro, Brazil 